\title{
Mobile Surface Reflectometry
}

\author{
J. Riviere $^{1}$, P. Peers ${ }^{2}$, A. Ghosh ${ }^{1}$ \\ ${ }^{1}$ Imperial College London ${ }^{\dagger}$ \\ ${ }^{2}$ College of William \& Mary
}

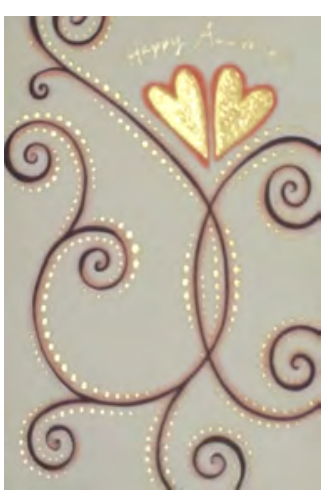

(a) Flash-based reflectometry

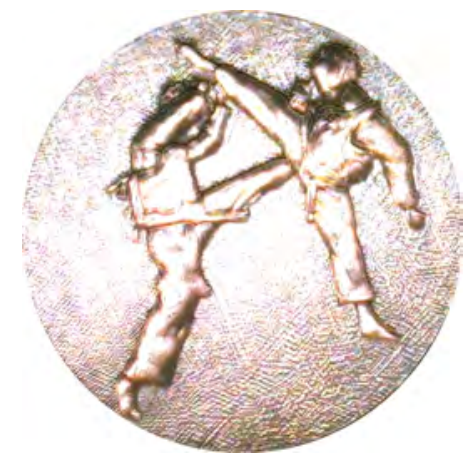

(b) LCD-based reflectometry

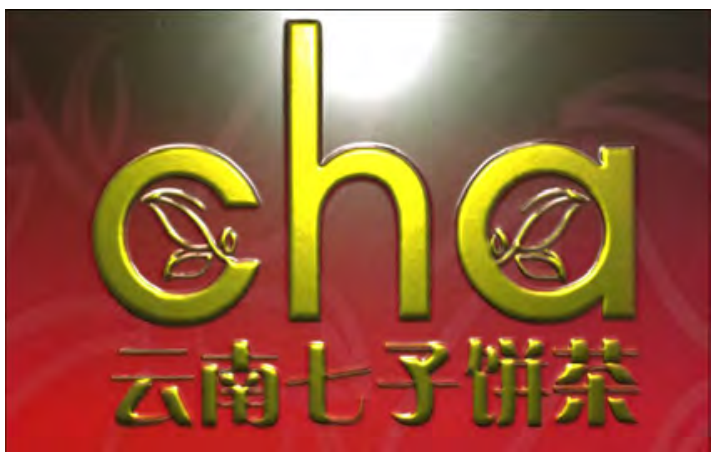

(c) Appearance transfer

Figure 1: Examples of surface reflectance recovered using mobile reflectometry: (a) A spatially varying rough specular material acquired using our handheld mobile flash-based reflectometry. (b) Highly specular surface reflectance recovered using mobile LCD-based reflectometry, with enhanced mesostructure from close-up observations under natural lighting. (c) Surface reflectance of a large spatial varying material sample recovered using appearance transfer under natural lighting from surface reflectance obtained using the LCD-based approach for a small reference patch.

\begin{abstract}
We present two novel mobile reflectometry approaches for acquiring detailed spatially varying isotropic surface reflectance and mesostructure of a planar material sample using commodity mobile devices. The first approach relies on the integrated camera and flash pair present on typical mobile devices to support free-form handheld acquisition of spatially varying rough specular material samples. The second approach, suited for highly specular samples, uses the LCD panel to illuminate the sample with polarized second order gradient illumination. To address the limited overlap of the front facing camera's view and the LCD illumination (and thus limited sample size), we propose a novel appearance transfer method that combines controlled reflectance measurement of a small exemplar section with uncontrolled reflectance measurements of the full sample under natural lighting. Finally, we introduce a novel surface detail enhancement method that adds fine scale surface mesostructure from close-up observations under uncontrolled natural lighting. We demonstrate the accuracy and versatility of the proposed mobile reflectometry methods on a wide variety of spatially varying materials.

Categories and Subject Descriptors (according to ACM CCS): I.3.7 [Computer Graphics]: Three Dimensional Graphics and Realism-Color, shading, shadowing, and texture. I.4.1 [Image Processing and Computer Vision]: Digitization and Image Capture-Reflectance.
\end{abstract}

Keywords: SVBRDF, mobile device, reflectometry, 2D/3D tracking, registration.

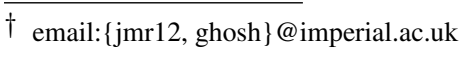

(c) 2015 The Author(s)

Computer Graphics Forum (c) 2015 The Eurographics Association and John

Wiley \& Sons Ltd. Published by John Wiley \& Sons Ltd.

\section{Introduction}

Modeling the appearance of real-world materials is an active area of research that has impacted a wide range of 

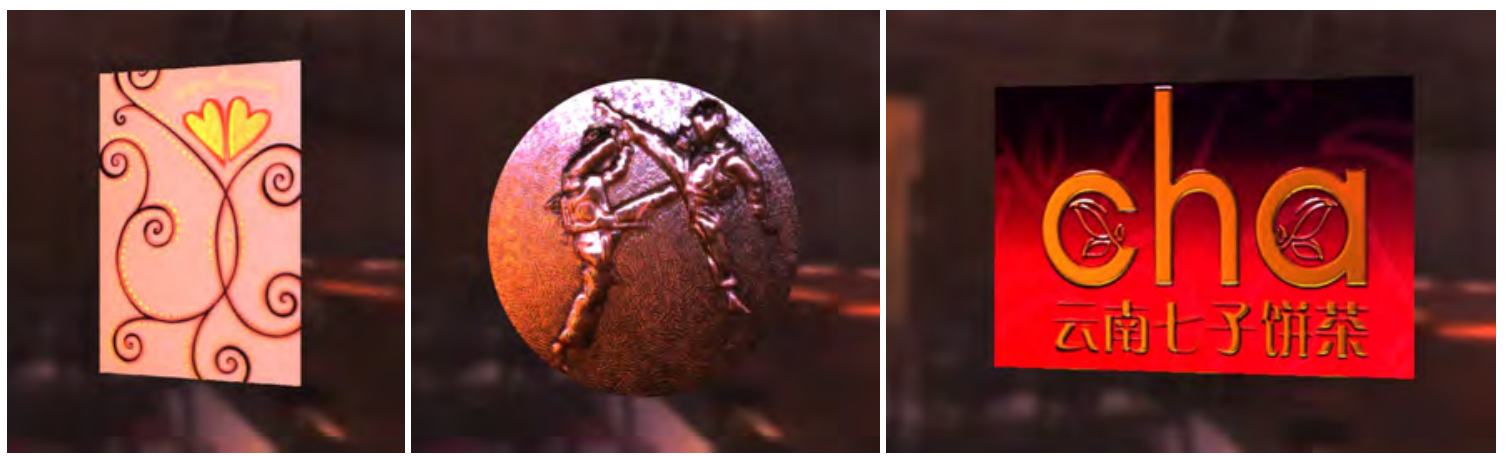

Figure 2: Acquired samples from Fig. 1 rendered in the Grace Catheral environment.

applications, such as visual effects, computer games, virtual reality, cultural heritage, product design, and advertising. Driven by the advances in digital imaging technology, measurement has become an ubiquitous and integral component in appearance modeling of real-world materials, especially for materials that exhibit rich variation in appearance which would be hard to model procedurally or artistically [MGW01,GTHD03,GCP*09,RWS*11,AWL13]. A recent and emerging trend in measurement-based appearance modeling is to make acquisition and modeling more practical and more accessible for the user.

This paper furthers the goal of making the acquisition of rich spatially varying isotropic surface reflectance and mesostructure of planar material samples more accessible to non-expert users by using commodity mobile devices such as tablets and cell phones. However, unlike prior work that uses separate devices for measurement and lighting (e.g., a camera-linear light source pair [RWS*11], or a camera-LCD panel pair [GCP*09, AWL13]), our solution is truly off-theshelf, relying only on the mobile device to both control the incident lighting and record the resulting reflectance. We propose two distinct techniques for appearance acquisition using mobile devices, and also present a practical solution to address the inherent hardware limitation of mobile sensors:

- Mobile Flash-based Acquisition (Section 3). We exploit the near coaxial configuration of flash and backfacing camera on consumer mobile devices to acquire SVBRDFs with rough specular materials. We propose a free-form handheld acquisition strategy and explore two alternate approaches for estimating the incident lighting directions (and thus view direction).

- Mobile LCD-based Acquisition (Section 4). We exploit a tablet's LCD display to act as an extended light source to enable accurate acquisition of highly specular SVBRDFs. To model the surface reflectance of large material samples, we propose a novel method to transfer surface reflectance from a small representative portion of the material sample captured under controlled LCD lighting to the entire sample observed under natural lighting.

- Surface Detail Enhancement (Section 5). We address the limited capture resolution of mobile acquisition setups by enhancing the estimated surface albedo and normal maps based on close-up observations of the samples under natural lighting.

Besides these technical contributions, the central practical contribution of this work is the successful demonstration of SVBRDF acquisition of planar samples using commodity mobile devices with limited control over hardware functions. We demonstrate that our mobile reflectometry approaches can reproduce high quality rendering results for a wide range of spatially varying materials and illumination conditions (e.g., Figs. 1 and 2).

\section{Related Work}

A large body of prior work on reflectance capture focuses on improving the acquisition accuracy and on improving the time-complexity of the acquisition process (e.g., see [WLL*09]). Typically, these surface reflectance capture techniques rely on complex and custom acquisition setups, only suited for laboratory-like environments. Recently, a new trust of research has emerged that addresses the accessibility of reflectance acquisition for non-expert users. We can roughly classify these novel methods in two categories: acquisition using commodity hardware, and freeform acquisition.

Commodity Hardware A common combination of commodity hardware components is a camera-LCD panel pair. Francken et al. [FCMB09] estimate surface normals and fit a Phong BRDF model to observations of an SVBRDF illuminated by a series of Gray codes. Ghosh et al. [GCP*09] employ spherical moments to estimate model-independent reflectance parameters. In addition to a Light Stage-based setup [MHP*07], Ghosh et al. also demonstrate surface reflectance acquisition with quadratic gradients using a camera-LCD panel pair. In subsequent work, Ghosh et al. [GCP*10] exploit the inherent polarization behavior of a camera-LCD panel pair to acquire surface reflectance of planar samples. Wang et al. [WSM11] estimate dual-scale properties of a homogeneous rough specular material from ob- 
servations under a single step-edge lighting condition. Aittala et al. [AWL13] demonstrate high-quality surface detail and surface reflectance acquisition by taking a small set of samples in the frequency domain. One of the two proposed reflectometry solutions also employs a camera-LCD panel pair. However, in contrast to prior work, we do not require the whole material sample to be illuminated by the LCD panel, which allows us to capture larger material samples and/or use smaller LCD panels. We also do not require a separate camera in our measurement setup.

Freeform Acquisition Masselus et al. [MDA02], and Winnemöller et al. [WMTG05] record fixed viewpoint reflectance fields by waving a light source around the scene. Both methods are geared towards image-based relighting, and do not fit compact surface reflectance models. Similarly, Chen et al. [CGS06] estimate mesostructure for shiny samples from observations of specular reflections from a hand waved light source. The proposed flash based acquisition also recovers diffuse and specular reflectance parameters in addition to surface mesostructure for a broad range of BRDFs.

Ren et al. [RWS*11] present a portable version of the linear light source reflectometer [GTHD03] where the linear light source is manually waved over the target sample. However, similar to Gardner et al., Ren et al. also require a separate fixed camera to observe the target scene. Chen et al. [CDP* $\left.{ }^{*} 14\right]$ extend linear light source reflectometry to anisotropic materials, and present a handheld version that integrates camera and light source in a single device.

Similar in spirit to the proposed mobile LCD panel-based acquisition, Dong et al. [DWT*10] perform a two-phase acquisition. A specialized device is used to capture detail surface reflectance of a moderate number of surface points, which are subsequently leveraged to upsample a sparsely sampled reflectance field obtained by waving a light source over the target.

All of the above methods either require specialized hardware, or employ a separate camera as part of the setup. In contrast, the proposed methods only require a single mobile device to record and sample the surface reflectance, thus providing a truly mobile solution with commodity hardware.

A final class of method estimate surface reflectance under uncontrolled lighting (e.g., [RZ10,LN12]), and thus only require a camera. However, these methods, in contrast to the proposed methods, are limited to spatially homogeneous material samples, or rely on a simplified reflectance model to characterize spatial reflectance variation.

\section{Mobile Flash-based Acquisition}

Typical commodity mobile devices such as tablets and cell phones are equipped with an LED flash light located near the back facing camera, a near-coaxial setup that allows us to capture the backscatter surface reflectance in a single photograph. Such backscatter observations are well suited for fitting microfacet BRDF models [AP07]. In the proposed mobile flash-based reflectometry method, we use a very intuitive capture process to obtain such backscatter observations; the user manually moves the mobile device around and aims it at the sample while directly observing the reflectance data on the mobile device's front LCD screen as it is being captured by the device's back camera (Section 3.1). In order to fit these backscatter observations to a microfacet BRDF model (Section 3.4), we first need to recover the lighting/camera direction (Section 3.2) and the surface normals (Section 3.3).

\subsection{Acquisition}

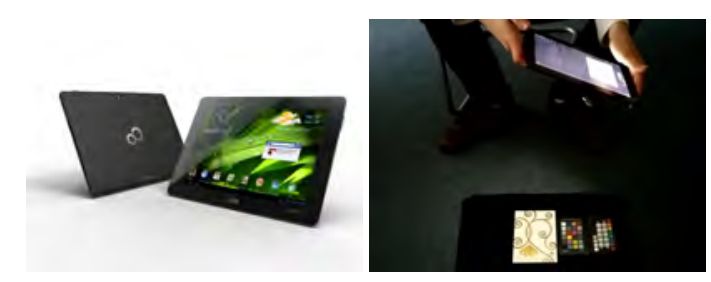

(a) mobile device

(b) hand-held acquisition

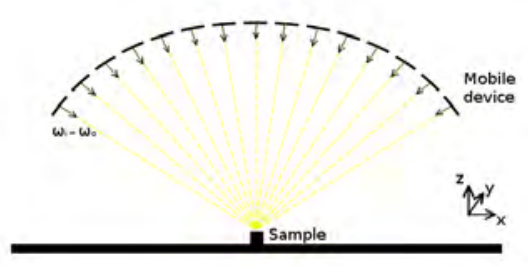

(c) flash capture

Figure 3: Mobile Flash-based Reflectometry. (a) Android tablet with 8MP back camera and flash illumination, employed for free-form reflectance acquisition (b). (c) Capture of dense backscattering measurements with flash illumination.

The acquisition process proceeds as follows: The user points the mobile device's back camera (and flash) at the planar reflectance sample from a distance of roughly $50 \mathrm{~cm}$ above the sample and then proceeds to capture a video sequence while sequentially capturing data from several directions over the upper hemisphere (Fig. 3 b, c. and the accompanying video). We limit ambient light levels to about 30 - 40 LUX (e.g., dimly lit room) during capture to ensure sufficiently strong observations from the flash compared to ambient lighting. To establish a reference frame for tracking, we ensure that the first frame is viewing the sample directly from the top at normal incidence. For reflectance calibration of the handheld measurements and to account for variation in distance to the sample and camera's auto exposure, we 


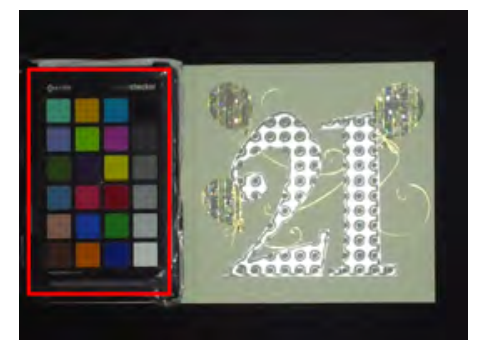

Figure 4: Free-form reflectance measurement calibration using an Xrite ColorChecker chart placed next to sample.

place an Xrite ColorChecker chart next to the sample during measurement and scale the measurements based on observed intensity variations in the diffuse gray squares of the color chart (Fig. 4).

In our experiments, we employ a Fujitsu Stylistic M532 10" Android tablet which has an 8MP back camera and LED flash (Fig. 3.a). We acquire reflectance data using the back camera at 1080p HD resolution available on the device for video capture. However, reflectance data acquired in the standard video capture mode suffers from compression artifacts. Instead, we grab uncompressed frames from the video preview during the data capture, and radiometrically linearize the grabbed LDR frames. Radiometric calibration is further complicated by the lack of exposure control on the acquisition device. Instead, we employ an Xrite ColorChecker chart and use a gamma-curve fitted to the known reflectance of the gray squares as the camera response function. We set the capture rate to 15 FPS - the limit for frame grabbing at full HD resolution on our device. A typical capture sequence lasts about 15 seconds and around 250 frames are recorded of the sample from several surrounding viewpoints.

We register the captured frame to the canonical frontal viewpoint using a combination of sparse feature tracking and optical flow. We do not rely on the detection of the ColorChecker chart as not all color checkers are guaranteed to be visible for all frames during a typical hand held capture sequence. Instead, we detect and track sparse 2D features [HS88] across the sequence of frames captured with flash illumination and compute homography matrices for $2 \mathrm{D}$ projection of all the frames to the first canonical frame (captured at normal incidence). We further refine the registration using a dense optical flow [LK81] based image warping.

\subsection{Lighting/view direction estimation}

A critical component in our mobile flash-based reflectometry method is the estimation of the lighting/view direction. We propose two alternative methods: sensor-based tracking and 3D tracking.

Sensor-based Tracking Typical mobile devices contain a

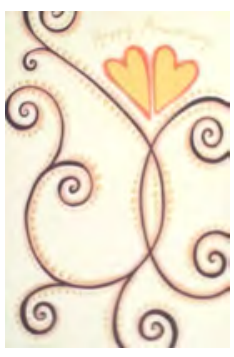

(a) diff. albedo

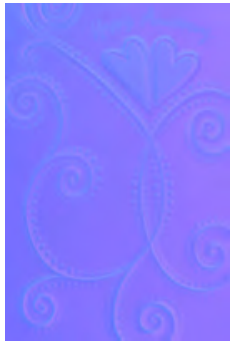

(c) surface normal

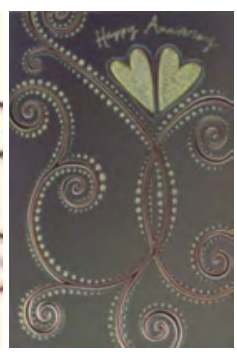

(b) spec. albedo

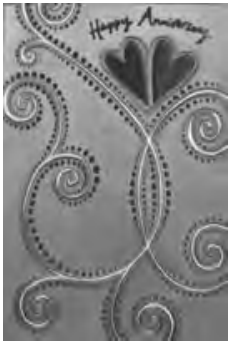

(d) spec. roughness
Figure 5: Estimated surface reflectance maps of a rough specular sample free-form acquired with mobile flash-based reflectometry.

wide variety of sensors that can aid in estimating the relative camera view/light direction. Specifically, we employ the magnetometer and accelerometer to estimate the orientation of the device with respect to a reference coordinate system. We initialize the reference coordinate frame with respect to the sample placed on the X-Y plane, and with the first frame (by convention acquired at normal incidence) corresponding to view/light direction along $+\mathrm{Z}(\theta=0, \phi=0$ in spherical coordinates). With this parameterization, we interpret changes in the readings of the internal sensors due to change in device orientation as change is $\theta$ and $\phi$ to obtain the view/light directions for each acquired frame.

3D Tracking Alternatively, when the mobile device lacks the required sensors and/or the material sample exhibits sufficient texture, 3D tracking can be used to estimate the lighting/view direction. Inspired by the recent success of PTAM [KM07] for unstructured light fields [DLD12] and for augmented reality with surface light fields [JND12], we estimate the camera parameters (both internal and external) using PTAM. While limited to sufficiently rich textured material samples, 3D tracking provides the 3D position of the camera (and thus light source) as opposed to a 2D direction with the sensor based approach. This allows us to take local lighting effects and camera perspective in account, producing more accurate reflectance estimates.

\subsection{Normal Estimation}

While our observations are ideally suited for classical photometric stereo [Woo80], prior work [MHP*07] has shown that specular reflections better reflect the "true" surface shape. 


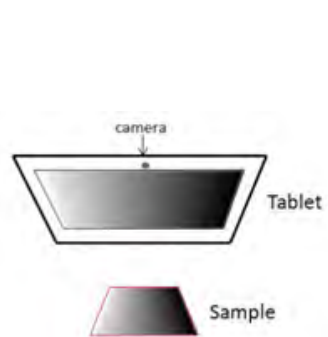

(a) LCD setup

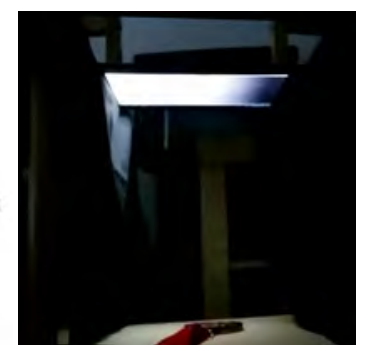

(b) acquisition
Figure 6: Measurement setup for highly specular sample using a tablet's LCD illumination.

Key to exploiting such specular cues is the observation that both the diffuse and specular reflectance peaks when the backscattered direction aligns with the surface normal. However, due to the discrete sampling, the exact normal direction might not have been sampled. We therefore compute the surface normal of each surface point as the weighted average of the brightest reflection directions. To give more importance to samples close to the surface normal direction, we weight the samples by their corresponding observed intensity. Figure 5.c shows an example of a recovered normal map with this approach.

\subsection{BRDF fitting}

We estimate the diffuse albedo as the median of the measured intensities after discarding the top $20 \%$ bright samples and factoring in the cosine foreshortening. We then estimate the specular albedo as the hemispherical integral of the diffuse subtracted measurements. Finally, we fit the observed backscattering profile [AP07] to a microfacet BRDF model to obtain per pixel specular roughness for rendering (Fig. 5). In particular we use the GGX distribution [WMLT07] to model the microfacet normal distribution.

\section{Mobile LCD-based Acquisition}

The previous mobile flash-based reflectometry method samples the surface reflectance from a set of discrete directions. As with other sample-based methods, this places a limit on the sharpness of the specular surface reflectance that can be accurately recovered. We propose an alternate mobile reflectometry solution for sharp specular materials that utilizes the mobile device's LCD panel as an extended illumination source. However due to the small size of the LCD panel and the off-center location of the front camera, only a small $5 \mathrm{~cm}$ $\times 5 \mathrm{~cm}$ section of the material sample can be directly recovered (Section 4.1). We rely on a novel appearance transfer method to extend the acquired surface reflectance to larger material samples (Section 4.2).

\subsection{Direct Reflectance Estimation}

Acquisition We recover the surface reflectance from observations of the material sample from the front camera and il-

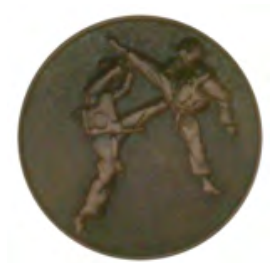

(a) diff. albedo

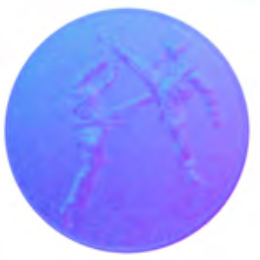

(c) surface normal

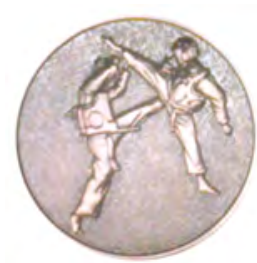

(b) spec. albedo

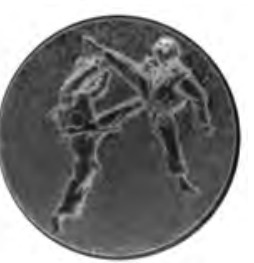

(d) spec. roughness
Figure 7: Surface reflectance maps of a highly specular sample estimated from mobile LCD-based reflectometry measurements.

luminated by the LCD-panel. The mobile device is statically mounted at a distance of $45 \mathrm{~cm}$ above the sample facing the sample at normal incidence (Figure 6). We exploit the inherent linear polarization of the LCD panel for diffuse-specular separation, and capture the sample twice for each lighting condition with a differently oriented linear polarizer filter in front of the camera (90 degree rotated). We attach a regular plastic sheet linear polarizer in front of the camera which proved sufficient for our purposes and also simplified the mounting. ${ }^{\dagger}$ As in Ghosh et al. [GCP* 09 ], we illuminate the sample with a constant lighting pattern, linear gradients, and axis aligned quadratic gradients.

Similarly as for the flash-based solution, we employ a Fujitsu Stylistic M532 10" Android tablet, but use the 2MP front camera. We empirically determined that the axis of polarization of the LCD panel is 45 degrees, and achieve parallel and cross polarization on the camera by mounting a linear polarization filter at 45 and 135 degrees respectively in front of the camera. Due to the limited light levels of the LCD screen, we restrict acquisition for this setup to very dimly lit room with $15-20$ LUX of ambient illumination.

\section{Reflectance Recovery}

First, we separate the observations into their diffuse and specular components using the parallel and cross-polarized input images. Next, similar to Ghosh et al. [GCP*09], we estimate albedo from the constant illumination images, the surface normals from the linear gradients, and specular rough-

\footnotetext{
$\dagger$ Glass polarization filters are now also becoming available for mobile cameras and are also very inexpensive. However, we currently only found circular polarizer glass filter attachments for photography applications which was not suitable for our device's LCD panel.
} 


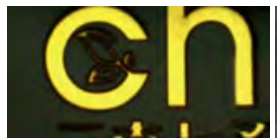

(a) spec. albedo

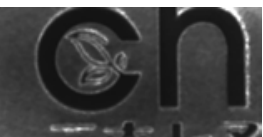

(b) spec. roughness
Figure 8: Specular reflectance of a reference patch of the "cha box" sample acquired our LCD-based solution.

ness from the quadratic gradients. Figure 7 shows recovered diffuse and specular reflectance and normal maps of a specular material.

\subsection{Appearance Transfer}

For larger samples, the LCD-based solution can only recover the surface reflectance of a small section of the material sample (Figure 8). However, we observe that many spatially varying materials are stationary in appearance, i.e., similar mixtures of materials occur on different sections of the sample (compare the full sample in Figure 1 to the captured section in Figure 8). This suggest that we can transfer the surface reflectance obtained with the LCD-based approach to other surface points.

Acquisition To guide the transfer, we require surface normal estimates of each surface point and a significant specular response at each surface point.

We use the mobile flash-based reflectometry approach proposed in Section 3 to estimate the surface normals (Figure 9.b). In addition, the flash-based reflectometry approach also provides us with reliable diffuse reflectance estimates for all surface points (Figure 9.a). Furthermore, because the back camera has a higher resolution than the front camera (8MP vs $2 \mathrm{MP}$ ), we also obtain a more detailed normal and diffuse reflectance maps.

The sample density of the flash-based solution is too low to provide a specular response at each surface point for highly specular materials. We resolve this issue by taking two additional photographs of the sample illuminated by a commonly occurring natural lighting condition, namely natural outdoor illumination being cast through a window into a room. Essentially, the window serves as an extended light source similar in purpose to the LCD panel. We capture two photographs: one at normal incidence, and one at a more grazing angle towards the window (at approximately $65^{\circ}$ incidence) (Figures 9.c and 9.d). We opt for this particular lighting condition and viewpoints to isolate specular cues: the key idea is that the photograph at grazing angle has strong specular reflections due to mirror reflection from the window, while the photograph at normal incidence is diffuse dominated due to hemispherical integral over the indoor illumination. To isolate the specular cues, we first align the grazing angle photograph to the normal incidence photograph, and subsequently subtract the latter from the aligned image.

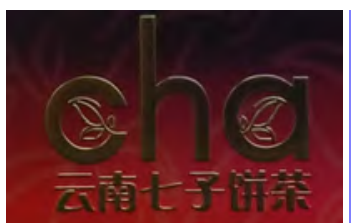

(a) diff. albedo

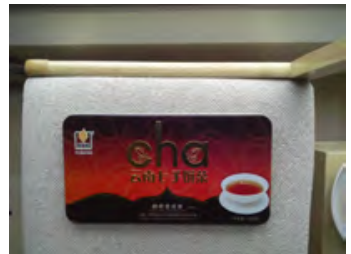

(c) normal incidence

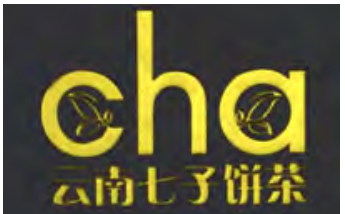

(e) spec. albedo

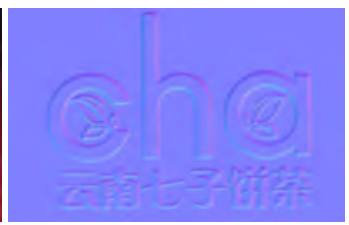

(b) surface normal

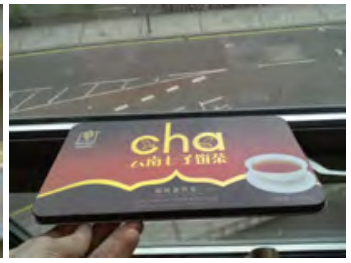

(d) grazing angle

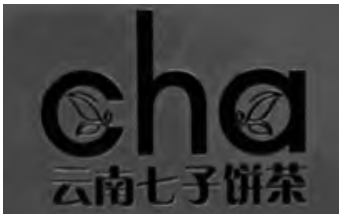

(f) spec. roughness
Figure 9: Top: Diffuse albedo and surface normal estimated using our flash-based approach. Center: Observations of the entire large specular sample lit with uncontrolled natural illumination - predominantly diffuse indoor illumination at normal incidence and predominantly outdoor illumination at grazing angle. Bottom: Transferred specular reflectance parameters to the entire sample from the smaller measured section shown in Fig. 8.

Note that we employ natural illumination seen through a window as a large extended light source for exciting specular reflections for specular transfer. We assume that illumination is fairly constant within the limited solid angle of the specular lobe. This condition is commonly satisfied with fairly even outdoor illumination. However, this assumption could break if the window is illuminated by high frequency illumination such as sun rays breaking through clouds or tree branches causing occlusions.

Transfer To transfer the reflectance from the representative patch to the rest of the sample, we build two intensitybased look-up tables (LUTs) for the corresponding sections of specular cues under natural lighting, and the estimated specular albedo and roughness obtained from LCD-based direct reflectance estimation. Transferring the reflectance is now simply an intensity-based look-up to the corresponding albedo and roughness. We employ 4096 LUT bins for quantizing the intensity range. Since not all bins have entries, we fill missing values using linear interpolation during the appearance transfer. An added advantage of using a LUT is that measurement noise is averaged out to a great extent. The final transfer result is shown in Figures 9.e and 9.f. 


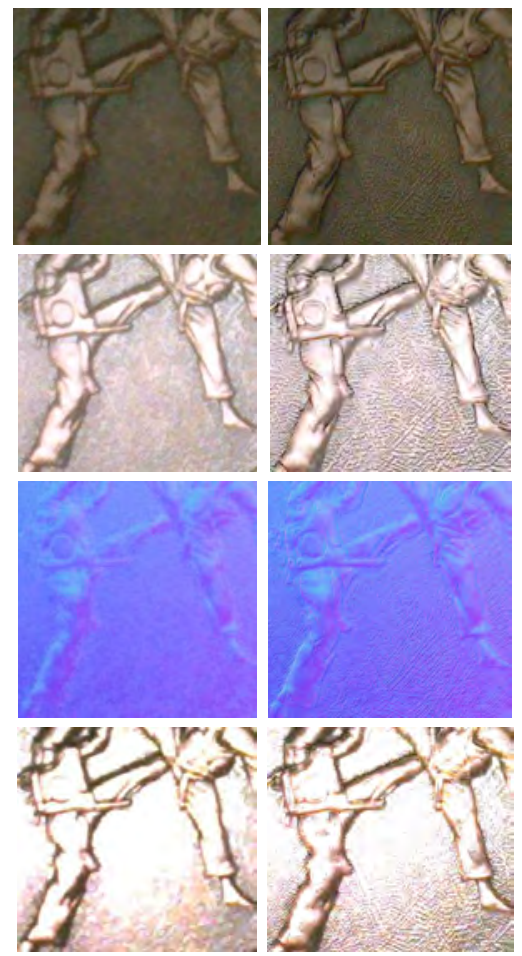

(a) acquired

(b) enhanced

Figure 10: Surface detail enhancement of reflectance maps of a specular sample. Left-column: Direct capture with mobile LCD-based reflectometry. Right-column: Surface detail enhancement. Top to bottom: diffuse albedo, specular albedo, surface normal, and rendering.

\section{Surface Detail Enhancement}

The presented mobile flash-based and LCD-based reflectometry methods are limited by the capture resolutions of the acquisition setups. Although the flash-based capture employs a high resolution $8 \mathrm{MP}$ back camera, it is restricted to a $1080 \mathrm{p}$ resolution for video mode capture. Furthermore, the sample is imaged from a distance to observe both the sample and (a portion of) the ColorChart. Similarly, the LCD-based is limited by the 2MP front camera, and requires the sample to be placed at a reasonable distance from the tablet further limiting the size of observable fine scale mesoscopic surface details.

Inspired by the mesoscopic augmentation step proposed by Beeler et al. $\left[\mathrm{BBB}^{*} 10\right]$ for facial geometry reconstruction, we propose to alleviate the limited resolution of surface details by augmenting not only the surface normals (as in Beeler et al.) but also diffuse and specular albedo. Similar to the proposed appearance transfer, we make two observations of the sample in a room illuminated by natural outdoor illumination through a window: one at normal incidence exciting mostly diffuse reflectance; and another closer to graz- ing incidence with the sample facing the window to excite specular reflectance. We employ the 8MP back camera and capture the two images from close-up viewpoints to image the entire sample at the highest possible resolution, revealing additional mesoscopic surface details not visible in the reflectance measurements. To transfer the detailed mesostructure, we add the high pass filtered details from the high resolution image captured at normal incidence to the diffuse albedo map. Similarly, high pass filtered details from the high resolution image captured closer to grazing incidence, are added to the specular albedo map. Finally, we augment the surface normal map by adding the $\mathrm{X}$ and $\mathrm{Y}$ gradients of the high resolution image (captured at approximately grazing angle) to the $\mathrm{X}$ and $\mathrm{Y}$ components of the surface normal (with appropriate scaling and renormalization).

To transfer the detailed mesostructure, we follow a coarseto-fine approach to register the lower resolution reflectance maps and high resolution cue photographs. We start by downsampling the cue photographs to the native resolution of the reflectance maps and separate both sets of maps into their low and high frequency components using a high pass filter with a Gaussian of 4 pixels width. We then register the corresponding high frequency components using a SIFT feature matching [Low04] based approach with outlier pruning (RANSAC) to find the best affine transformation for the registration. The aligned high frequency details from the cue photographs are then added to the low frequency components of the corresponding maps and the same steps are iterated at higher resolutions (two levels with doubling of resolution at each level) until we reach the native resolution of the cue photographs.

Similar to Beeler et al., the enhanced details are not exact, but provide plausible surface details in both the diffuse and specular albedo maps and the surface normal map obtained with direct capture for rendering purposes (see Fig. 10 and accompanying video).

\section{Analysis and Results}

Qualitative Validation Figure 11 compares reference photographs of the material samples shown in Figure 1 illuminated by a single "point" light with renderings of recovered surface reflectance. The "Greeting card" example (Figure 11, top) was acquired using mobile flash-based reflectometry; the "medal" example (Figure 11, center) was captured with mobile LCD-based reflectometry and further processed with the proposed surface detail enhancement step; and the "Cha box" example (Figure 11, bottom) is the result of appearance transfer from a small reference patch acquired with mobile LCD-based reflectometry. Direct capture results were acquired indoors in a moderately darkened room for both flash and LCD measurements. Further processing with observations under natural lighting from a window was done for the "medal" and "Cha box" samples. Overall, both renderings and reference photographs are a good qualitative 

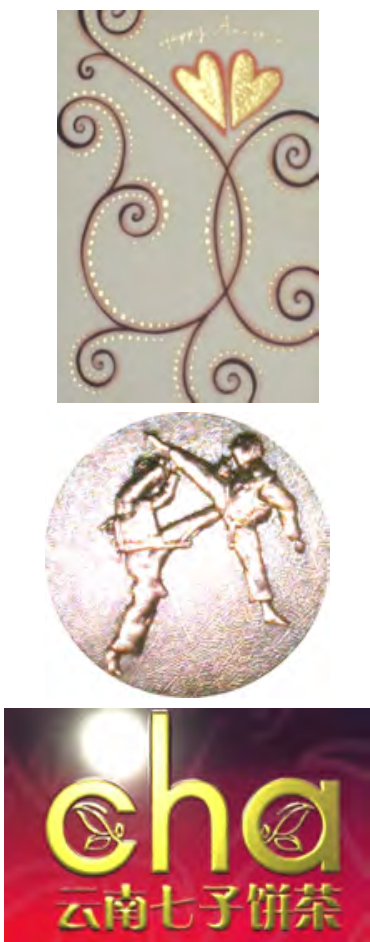

(a) rendering
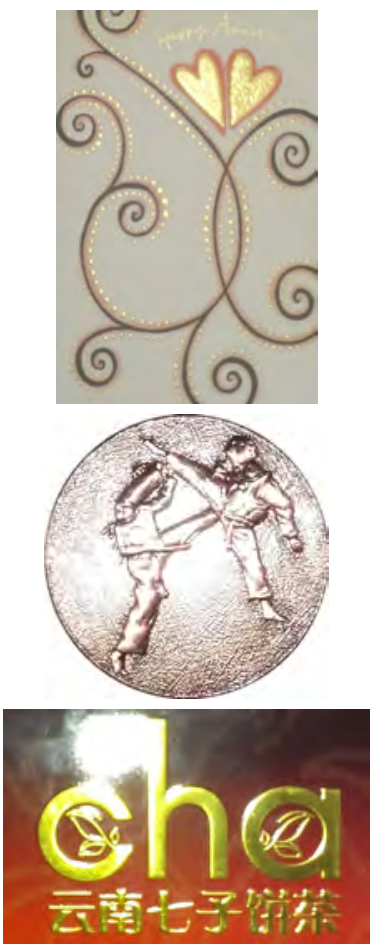

(b) reference photograph
Figure 11: Comparison of renderings with estimated reflectance parameters with reference photographs under point source illumination from near normal incidence. Top: Rough specular sample acquired using the flash-based approach. Center: Specular sample acquired with LCD-based reflectometry. Bottom: A larger sample reconstructed using appearance transfer.

match. Most of the differences can be explained by slight differences between view and lighting in the reference photographs and the renderings. The specular highlights on the "Cha box" are less strong in the rendering due to the limited dynamic range of the tablet's front camera.

Figure 17 shows recovered reflectance and normal maps of two additional flash-based (top and center) and one additional LCD-based reflectometry results (bottom). Here, the presented albedo and normal maps include the surface detail enhancement step described in Section 5. Figure 18 shows visualizations of appearance transfer, renderings and a reference photograph of a large book cover. Note how rendering with a directional light source incident near grazing angle highlights the specular areas (also see accompanying video). Finally, Figure 12 shows additional examples of renderings compared to photographs under novel combinations of view and light directions which are much more oblique and not employed for reflectance measurements. Note that these examples depict oblique mirror angle reflections while the measurements purely involve backscattering directions

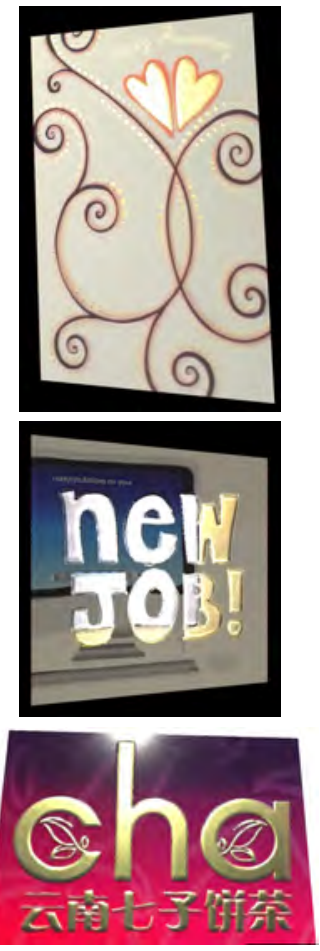

(a) rendering
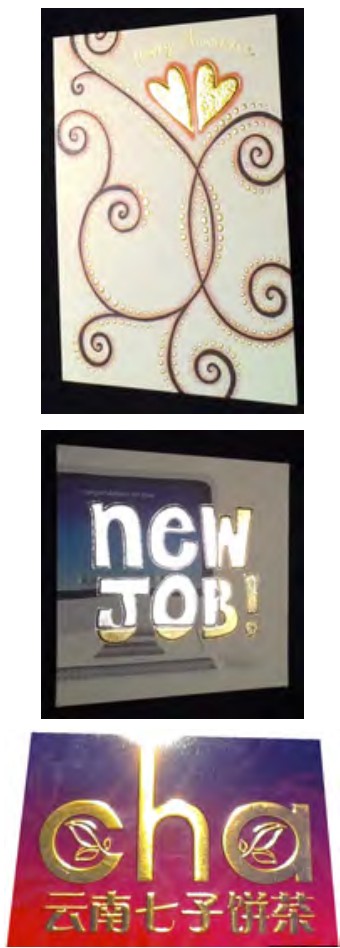

(b) reference photograph
Figure 12: Comparison of renderings with reference photographs under point source illumination for novel camera view - light direction combinations not employed for reflectance capture. Top and Center: Rough specular samples acquired using the flash-based approach. Bottom: Large specular sample acquired with $L C D$-based reflectometry followed by appearance transfer.

closer to normal incidence. These results demonstrate that the proposed mobile reflectometer solutions can attain good qualitative matches for material samples exhibiting a wide range of spatially varying surface reflectance.

Accuracy of Roughness To validate the accuracy of the estimated specular roughness parameters, we compare the result from our mobile flash-based reflectometry approach to estimates obtained with the method proposed by Ghosh et at. [GCP* ${ }^{*}$ 09] (Figure 13). The majority of differences in results are due to differences in the acquisition setups and procedure. For the method of Ghosh et al. [GCP*09], we employ a 24" LCD monitor and a Canon EOS 650D DSLR. With exception of the lower camera resolution and slight blur (due to the handheld nature of the flash based acquisition procedure), the obtained parameter maps are a good qualitative match. A quantitative comparison between the roughness maps produced by the two methods also shows very minor differences of $1.9 \%$ and $2.4 \%$ RMSE respectively for the top and bottom samples. Since our LCD-based 

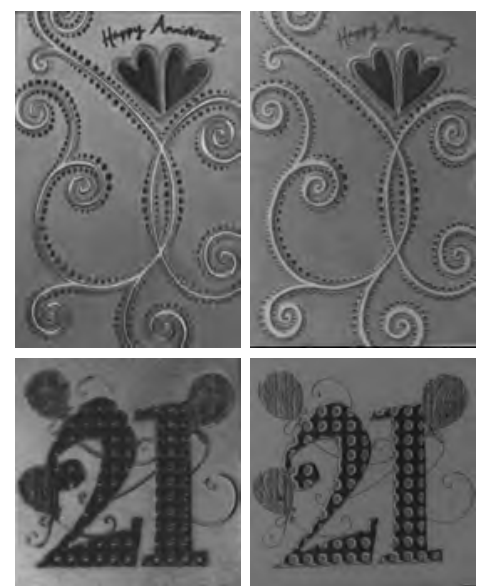

(a) free-form capture

(b) LCD monitor

Figure 13: Comparison of specular roughness maps obtained with free-form flash-based reflectometry observations and with the approach of Ghosh et al. [GCP* 09].

solution relies heavily on Ghosh et al.'s method, we believe that the results are in principle very similar to that of Ghosh et al. except for the limiting factors of lower camera resolution and sensitivity of the front camera on the mobile device.

Surface Normal Accuracy We compare the results of surface normal estimation obtained with the two variants of light/view directions (Section 3.2). On average, a $6^{\circ}$ difference is observed between sensor-based and 3D tracking on rough specular materials. A key difference between 3D tracking and sensor-based tracking is that the latter assumes directional lighting, ignoring spatial differences in incident lighting directions. To better understand the contribution of the assumption that the lighting is directional, we also compute the average normal error from directional light directions set to the normalized 3D tracked light direction. In this case, the average difference reduces to $4^{\circ}$, indicating that normal estimation using the internal sensors of the mobile device are quite accurate. We note that despite some difference in surface normal estimation with the sensor-based method compared to 3D tracking, the specular roughness maps and corresponding renderings are very comparable with both approaches for rough specular samples (Fig. 14). We measured a minor 3.6\% RMSE difference between the roughness maps obtained with the two methods for the greeting card sample (Fig. $14 \mathrm{c}$, absolute error visualized in false color).

Radiometric Calibration Accuracy We validate the accuracy of the radiometric calibration obtained from fitting a gamma-curve to an Xrite ColorChecker chart (placed next to the sample for the flash based capture, or separately captured for the LCD panel setup), by comparing the measured values of the gray square of the Xrite ColorChart (captured with the tablet's back camera and flash illumination) with the ground

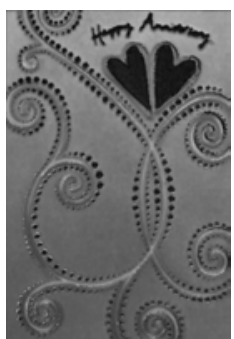

(a) sensor-based

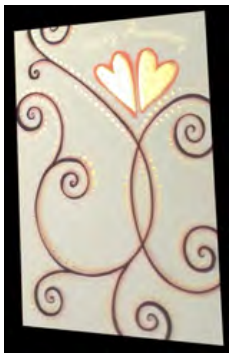

(d) sensor-based

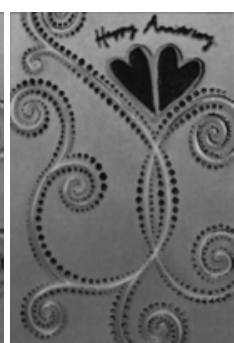

(b) 3D tracking

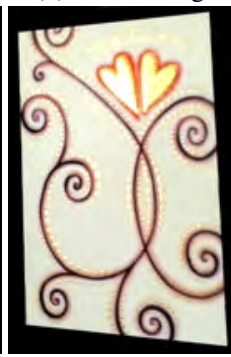

(e) 3D tracking

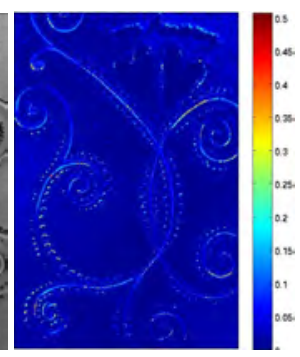

(c) $=\mid \mathrm{a}-\mathrm{b}$

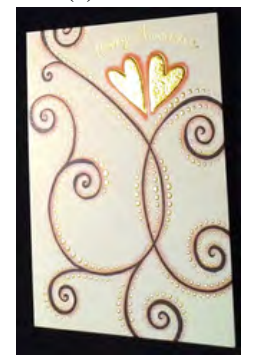

(f) photograph
Figure 14: Comparison of specular roughness maps (toprow) and renderings (bottom-row) obtained with sensorbased tracking versus $3 D$ tracking with PTAM.

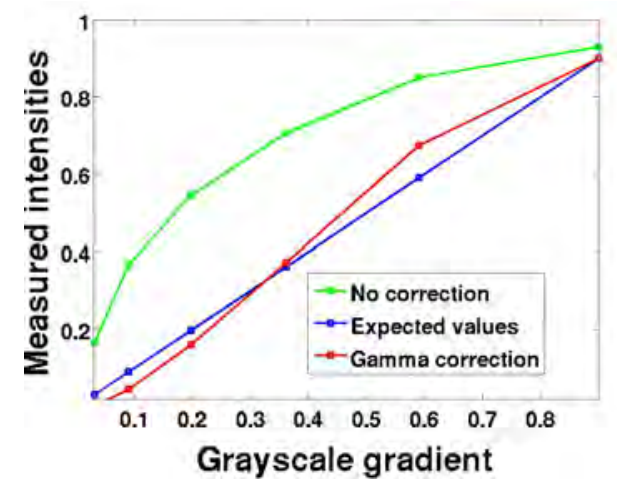

Figure 15: Linearization of measured intensities using a Gamma curve. The six plotted values correspond to the intensities of the gray squares on an Xrite ColorChart.

truth brightness values of each square. As seen in Figure 15, the measured values of the gray squares after a Gamma (3.2) correction are a close match to the true values.

3D Tracking vs Sensors PTAM based 3D tracking is slightly more accurate than the sensor-based estimation of view and light parameters. However, 3D tracking works best for materials samples with significant texture such as the greeting card in Fig. 1, a. However, PTAM is very sensitive to tracking errors due to lack of textures, strong specularities or motion blur. In such cases (e.g., the samples in Fig. 17 which are dominated by specular areas), only a few 


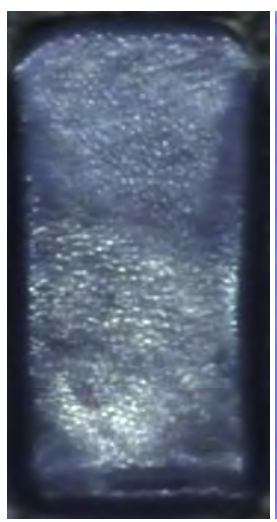

(a) Phone cover

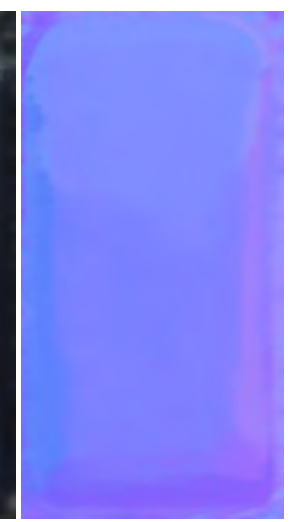

(b) normal map
Figure 16: Failure case for the flash-based free form acquisition approach. Here, the fine scale mesostructure on the dark blue phone cover is not resolved in the estimated surface normal map due to the distance of the mobile camera to the sample and lack of sub-pixel alignment due to the low albedo texture of the phone cover.

frames can be robustly tracked providing insufficient information for accurately fitting of per pixel BRDFs. For these samples we employed sensors based light/view estimation.

\section{Discussion}

Despite the less controlled acquisition procedure, the proposed mobile reflectometry approaches achieve qualitative good results. However, a number of limitations remain:

- The free-form acquisition process in the flash-based approach exhibits a slight blur in the reflectance maps due to minor misalignments and minor motion blur in the acquired multi-view data. Furthermore, only a limited number of lighting directions are sampled, limiting the method to rough specular materials. Fine scale surface details may not be fully resolved in some cases due to distance of the mobile camera from the sample. The quality of tracking can also suffer a bit for surfaces with low albedo and uniform texture, causing blurring of fine scale features. Fig. 16 shows an example of this for a dark blue leather phone cover where fine scale surface details are not resolved in the surface normal map due to lack of sub-pixel alignment of the acquired data. This can be alleviated to some extent with further processing with the surface detail enhancement step.

- The quality of the direct LCD-based reflectometry solution is limited by the low resolution and limited dynamic range of typical mobile device's front cameras, and by the limited overlap between the camera and LCD. The former can be alleviated with the surface detail enhancement step while the latter is alleviated by the proposed appearance transfer method.
Many of the above limitations are a direct result of the limitation imposed by existing camera technology on mobile devices. We expect further improvements with future evolutions of mobile sensors.

\section{Conclusion and Future Work}

In this work we introduced two novel approaches for mobile surface reflectometry. We proposed a handheld flashbased solution that exploits the near co-axial configuration of camera and flash on mobile devices, and which is suited for rough specular materials. For highly specular materials, we instead propose to employ extended illumination from a tablet's LCD screen in conjunction with the front camera to acquire surface reflectance properties, and exploit the inherent polarization of LCD screens to separate diffuse and specular reflectance. We alleviate the impact of the limited overlap of the front camera's coverage and the LCD-panel's illumination with a novel appearance transfer method that combines controlled measurement of an exemplar section from the full material sample with observations of the entire sample under natural lighting. We employ similar observations under natural lighting to enhance fine scale surface details. We demonstrated that the proposed mobile reflectometry solution achieves high quality reflectance and mesostructure reconstructions on a wide range of planar material samples. For future research, we will investigate mobile reflectometry solutions under less restricted and unconstrained ambient lighting conditions, such as indoor condition with significant ambient lighting (i.e., greater than the 30 - 40 lux in our current experiments) and in outdoor conditions. It will also be very interesting to combine internal sensors with $3 \mathrm{D}$ tracking for a more robust tracking solution.

\section{Acknowledgements}

We take this opportunity to thank Jay Busch and Antoine Toisoul for assistance with the video, and Santa Ghosh and Saskia Mordijck for their support. This work was partially supported by an EPSRC First Grant EP/M00192X/1 and a Royal Society Wolfson Research Merit Award to Abhijeet Ghosh, NSF grants IIS-1217765 and IIS-1350323 to Pieter Peers, and Google Faculty Research Awards to both Ghosh and Peers.

\section{References}

[AP07] AShikHMin M., Premoze S.: Distribution-based BRDFs, 2007. URL: http://www.cs.utah.edu/ premoze/dbrdf/. 3, 5

[AWL13] Aittala M., Weyrich T., Lehtinen J.: Practical SVBRDF capture in the frequency domain. ACM Trans. Graph. 32, 4 (2013), 110:1-110:12. 2, 3

[BBB*10] Beeler T., Bickel B., Beardsley P., Sumner B., Gross M.: High-quality single-shot capture of facial geometry. ACM Trans. Graph. 29 (July 2010), 40:1-40:9. 7 
[CDP* 14] Chen G., Dong Y., PeERs P., Zhang J., Tong X.: Reflectance scanning: Estimating shading frame and BRDF with generalized linear light sources. ACM Trans. Graph. 33, 4 (August 2014). 3

[CGS06] Chen T., Goesele M., Seidel H.-P.: Mesostructure from specularity. In CVPR (2006), pp. 1825-1832. 3

[DLD12] DAVIS A., LEVOY M., DURAND F.: Unstructured light fields. Comp. Graph. Forum 31 (May 2012), 305-314. 4

[DWT*10] Dong Y., Wang J., Tong X., SNyder J., LAN Y., Ben-Ezra M., GuO B.: Manifold bootstrapping for SVBRDF capture. ACM Trans. Graph. 29, 4 (2010), 98:1-98:10. 3

[FCMB09] Francken Y., CUypers T., Mertens T., BEKAERT P.: Gloss and normal map acquisition of mesostructures using gray codes. In ISVC (2009), pp. 788-798. 2

[GCP*09] Ghosh A., Chen T., Peers P., Wilson C. A., DeBEVEC P. E.: Estimating specular roughness and anisotropy from second order spherical gradient illumination. Computer Graphics Forum 28, 4 (2009), 1161-1170. 2, 5, 8, 9

[GCP*10] Ghosh A., Chen T., Peers P., Wilson C. A., DeBEVEC P.: Circularly polarized spherical illumination reflectometry. ACM Trans. Graph. 29, 6 (2010), 162:1-162:12. 2

[GTHD03] Gardner A., Tchou C., Hawkins T., Debevec P.: Linear light source reflectometry. ACM Trans. Graph. 22, 3 (2003), 749-758. 2, 3

[HS88] HARRIS C., STEPHENS M.: A combined corner and edge detector. In Proc. of Fourth Alvey Vision Conference (1988), pp. 147-151. 4

[JND12] JaChnik J., Newcombe R. A., Davison A. J.: Realtime surface light-field capture for augmentation of planar specular surfaces. In Proc. ISMAR (2012), pp. 91-97. 4

[KM07] Klein G., Murray D. W.: Parallel tracking and mapping for small ar workspaces. In ISMAR (2007). 4

[LK81] LUCAS B., KANADE T.: An iterative image registration technique with an application to stereo vision. In Proc. International Joint Conference on Artificial Intelligence (1981), pp. 674679. 4

[LN12] LOMBARDI S., NISHINO K.: Reflectance and natural illumination from a single image. In ECCV (2012), pp. 582-595. 3

[Low04] LowE D. G.: Distinctive image features from scaleinvariant keypoints. International Journal of Computer Vision 60, 2 (2004), 91-110. 7

[MDA02] Masselus V., Dutré P., Anrys F.: The free form light stage. In Rendering Techniques (2002), pp. 247-256. 3

[MGW01] Malzbender T., Gelb D., Wolters H.: Polynomial texture maps. In Proceedings of the 28th annual conference on Computer graphics and interactive techniques (2001), SIGGRAPH '01, pp. 519-528. 2

[MHP*07] Ma W.-C., HaWkins T., Peers P., Chabert C.F., WeIss M., Debevec P. E.: Rapid acquisition of specular and diffuse normal maps from polarized spherical gradient illumination. In Rendering Techniques (2007), pp. 183-194. 2, 4

[RWS*11] REN P., WANG J., SNyder J., Tong X., Guo B.: Pocket reflectometry. ACM Trans. Graph. 30, 4 (2011), 45:145:10. 2, 3

[RZ10] ROMEIRO F., ZICKLER T.: Blind reflectometry. In ECCV (2010), pp. 45-58. 3

[WLL*09] Weyrich T., LaWrence J., Lensch H. P. A., RUSINKIEWICZ S., ZICKLER T.: Principles of appearance acquisition and representation. Found. Trends. Comput. Graph. Vis. 4, 2 (Feb. 2009), 75-191. 2

(c) 2015 The Author(s)

Computer Graphics Forum (c) 2015 The Eurographics Association and John Wiley \& Sons Ltd.
[WMLT07] Walter B., Marschner S. R., Li H., TORRAnCE K. E.: Microfacet models for refraction through rough surfaces. In Rendering Techniques (2007), pp. 195-206. 5

[WMTG05] Winnemöller H., Mohan A., Tumblin J., Gooch B.: Light waving: Estimating light positions from photographs alone. Comput. Graph. Forum 24, 3 (2005), 433-438. 3

[Woo80] Woodham R. J.: Photometric method for determining surface orientation from multiple images. Opt. Eng. 19, 1 (1980), 139-144. 4

[WSM11] Wang C.-P., Snavely N., Marschner S.: Estimating dual-scale properties of glossy surfaces from step-edge lighting. ACM Trans. Graph. 30, 6 (2011), 172:1-172:12. 2 

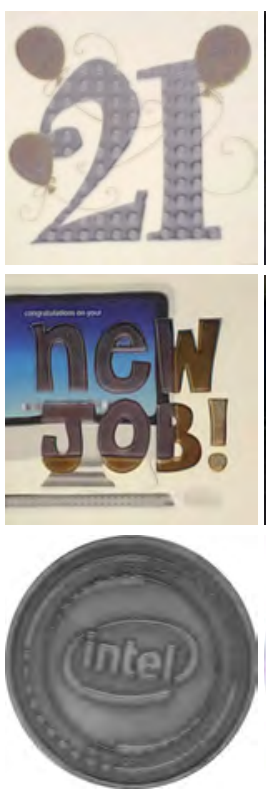

(a) diff. albedo
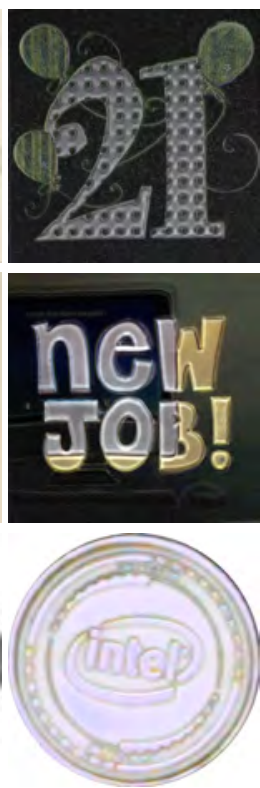

(b) spec. albedo
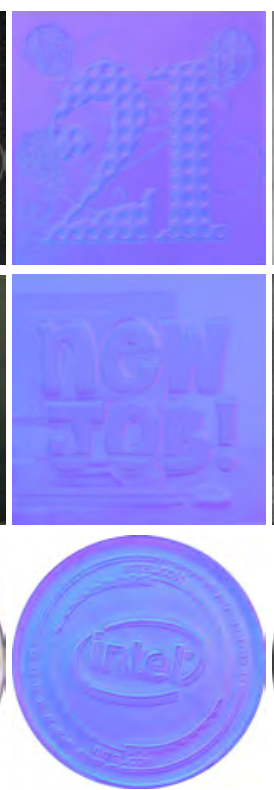

(c) surface normal
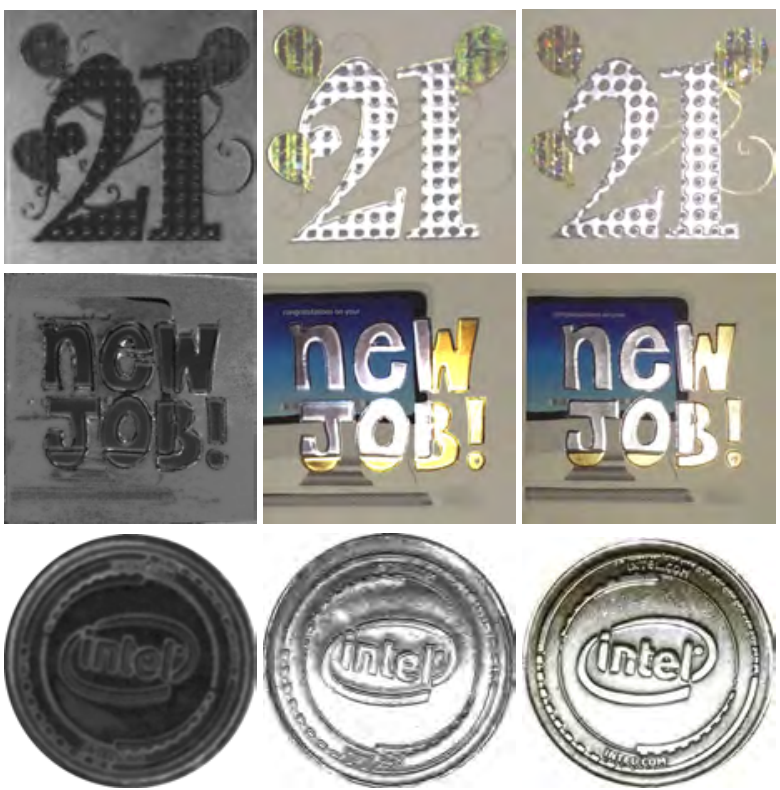

(d) spec. roughness
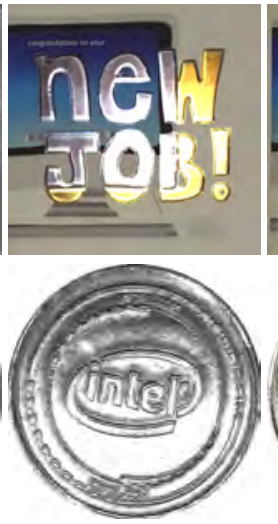

(e) rendering

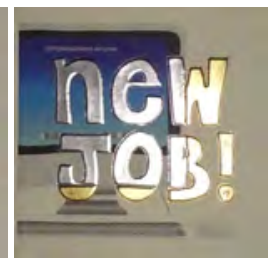

Figure 17: Reflectance properties acquired using a mobile device. Top and Center: Hand-held flash-based acquisition for rough specular material samples. Bottom: Mobile LCD-based acquisition results for highly specular BRDF.

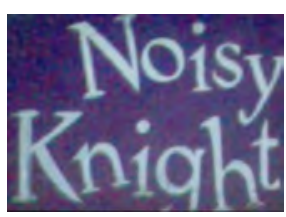

(a) spec. albedo

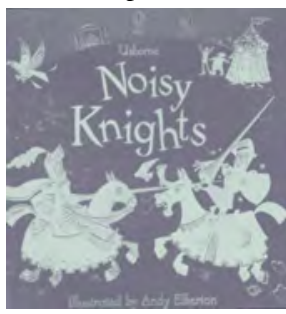

(g) transf. spec. albedo

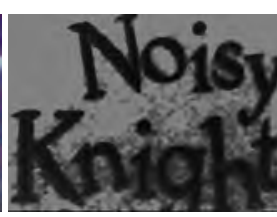

(b) spec. roughness

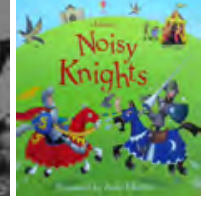

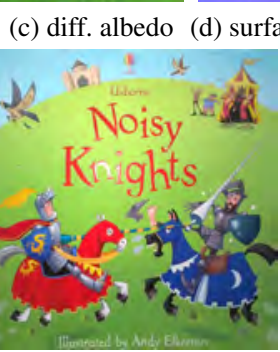

(i) rendering
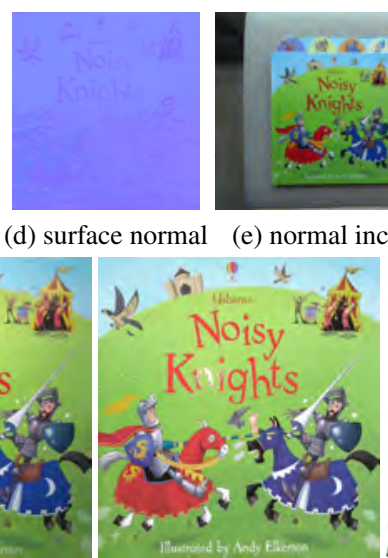

(j) photograph

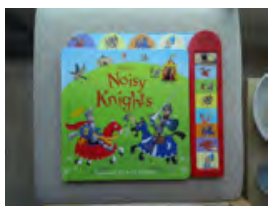

idence

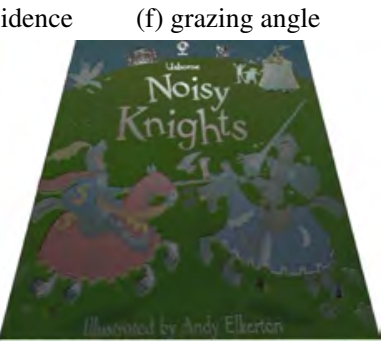

(k) grazing angle rendering

Figure 18: Reflectance properties of a book cover obtained using mobile LCD-based reflectometry and appearance transfer. $(\boldsymbol{a}-\boldsymbol{b})$ Specular reflectance properties of a reference patch acquired using the LCD-based approach. (c-d) Diffuse albedo and surface normals obtained with flash-based reflectometry. (e-f) Image used to guide the appearance transfer of the book cover captured at normal incidence and grazing angle and lit by natural illumination. $(\boldsymbol{g}$-h) Resulting specular reflectance maps for the entire sample obtained with appearance transfer. Rendering under a frontal point light source (i) is a good match to corresponding photograph $(j)$. ( $k$ ) Rendering with a directional light source near grazing incidence highlights the areas exhibiting strong specular reflectance. 